\title{
PERFIL CLÍNICO DAS MULHERES IDOSAS EM PÓS-OPERATÓRIO INTERNADAS EM CENTRO DE TERAPIA INTENSIVA
}

\section{CLINICAL PROFILE OF ELDERLY WOMEN IN POST-OPERATIVE HOSPITALIZED IN INTENSIVE CARE UNIT}

\begin{abstract}
Marina Serrano Vilalba, Fernanda Penido Matozinhos, Izabella Mendes de Souza Rocha, Thales Philipe Rodrigues da Silva, Flávia Sampaio Latini Gomes
\end{abstract}

Universidade Federal de Minas Gerais - UFMG

Abstract

The aim of this study is to identify the clinical profile of the elderly women from the intensive care unit in a public hospital in Belo Horizonte. It is a cross-sectional research. The sample consists of 53 elderly women. Data collection was performed in the electronic medical records of patients. The sample consisted of 53 elderly women. The median age was approximately 78 years ( \pm 11.0). The average time of hospitalization was of 6 days $( \pm 7.8)$. The main causes for hospital admission were: pertrochanteric fractures (32.1\%), cardiovascular diseases (24.5\%), neurologic (17.0\%) and infectious (9.4\%). Nine elderly women (20.9\%) had pressure ulcers. In relation to the outcome, 39 elderly women (73.6\%) were discharged to other sections, 12 (22.6\%) died and 2 (3.7\%) were transferred to other institutions. In recent years, advances have been made on the health of older women. This population group needs specific health policy plan and demands more differentiated care from the healthcare system and professionals.

Key words: Women's health; Health of the elderly; Nursing; Intensive Care Units; Health Profile.

\section{Resumo}

Objetivou-se identificar o perfil clínico das mulheres idosas provenientes do bloco cirúrgico, internadas no Centro de Terapia Intensiva de um Hospital Público de Belo Horizonte. Trata-se de uma pesquisa transversal. As coletas de dados foram realizadas por meio de prontuários eletrônicos das pacientes. A amostra consta de 53 mulheres idosas. A idade média foi de aproximadamente 78 anos $( \pm 11,0)$. O tempo médio de internação foi de 6 dias $( \pm 7,8)$. As principais causas da admissão hospitalar foram: fraturas pertrocantérica (32,1\%), doenças cardiovasculares (24,5\%), neurológicas $(17,0 \%) e$ infecciosas (9,4\%). Nove mulheres idosas (20,9\%) possuíam lesão por pressão. Das 53 mulheres idosas, 39 (73,6\%) receberam alta para outros setores, 12 (22,6\%) evoluíram para óbito e 2 $(3,7 \%)$ foram transferidas para outras Instituições. Nos últimos anos, avanços foram realizados sobre a saúde das mulheres idosas. Esse grupo populacional necessita de linhas de cuidados específicas nas políticas de saúde $e$ exige do sistema de saúde e dos profissionais cuidados cada vez mais diferenciados.

Palavras-chave: Saúde da mulher; Saúde do idoso; Enfermagem; Unidades de Terapia Intensiva; Perfil de Saúde. 


\section{Introdução}

Nas últimas décadas, a população brasileira tem passado por uma rápida transição epidemiológica, com mudanças nos padrões de fecundidade, morbidade e mortalidade ${ }^{1}$. Além disso, com os avanços na prevenção e tratamento de doenças, observa-se o aumento na expectativa de vida da população e, consequentemente, o crescimento da população idosa $^{2}$.

A Organização Mundial de Saúde (OMS) define a idade cronológica da fase idosa de acordo com o nível socioeconômico de cada país(3). Em países de baixa e média renda, ser idoso é considerado quando a idade cronológica é igual ou superior a 60 anos. Já em países de alta renda, tal idade cronológica aumenta para 65 anos ou mais ${ }^{3}$.

Segundo o último Censo Demográfico Brasileiro, realizado em 2010, o número de pessoas com 60 anos ultrapassou os 20 milhões, correspondendo a $11 \%$ da população brasileira e, destes, $55,5 \%$ eram mulheres e $44,5 \%$ homens $^{4}$.

No Brasil, o aumento da população idosa é marcada por uma feminização da velhice, resultado de maior expectativa de vida das mulheres idosas que, em média, vivem 8 anos a mais que os homens idosos 5 .

Dentre os fatores que contribuem para o processo de feminização da velhice destacam os fatores biológicos, em especial a proteção hormonal de estrógeno; a diferença de exposição aos fatores de risco de mortalidade; o uso/abuso de tabaco e álcool; as mortes violentas por assassinatos ou acidentes automobilísticos, cujas vítimas, em sua maioria são homens de faixa etária jovens - que, por causa da morte, não chegam na fase idosa da vida - e, também, à diferença de atitude em relação à saúde/doença, considerando que mulheres geralmente buscam mais os serviços de saúde - o que mostra maior preocupação com autocuidado ${ }^{6,5}$.

O perfil epidemiológico das mulheres idosas é bastante diferenciado das jovens e adultas. 0 envelhecimento está associado à diminuição das reservas fisiológicas e ao aumento do risco para desenvolvimento de doenças ${ }^{3}$. Quando ocorre um processo agudo de doença ou estresse, geralmente $o$ idoso tem menor capacidade fisiológica de superar as lesões, tornando-se mais vulnerável e frágil ${ }^{3}$ e exigindo mais recursos em saúde, principalmente pelo aumento das taxas de internação hospitalar dessa faixa etária ${ }^{7}$.

Conhecer o perfil de hospitalização torna-se importante, pois permite a elaboração de planejamento das ações em saúde para essa população especifica ${ }^{4}$.

Todavia, ressalta-se que a atenção à mulher idosa necessita de novos avanços na atenção à saúde. Observa-se que mesmo com a feminização da velhice, as taxas de doenças crônicas não transmissíveis são maiores entre os homens idosos do que em mulheres idosas (8). Entretanto, mulheres idosas são diagnosticadas precocemente, aumentando, consequentemente, a sobrevida e, portanto, levando a maior número de mulheres idosas internadas ${ }^{9}$.

Com o desenvolvimento da tecnologia científica, os procedimentos cirúrgicos têm sido cada vez mais empregados entre os pacientes idosos - para o diagnóstico e o tratamento de doenças - e, os Centros de Terapia Intensiva (CTI), cada vez mais utilizados no período pósoperatório $^{10}$.

O CTI é, portanto, um dos setores mais afetados com o fenômeno do crescimento do número de idosos, pois esses pacientes são mais susceptíveis a apresentar problemas crônicos de saúde, cuja agudização exacerba doenças que exigem complicados tratamentos, ocupando, assim, cada vez mais leitos dessas unidades ${ }^{11}$. 0 aumento da demanda de leitos por pacientes idosos sugere, portanto, uma preocupação dos profissionais de saúde no que se refere à assistência a esse grupo etário ${ }^{11}$.

Dessa forma, é adequado e necessário aprofundar a reflexão acerca da saúde da mulher idosa hospitalizada em setores específicos, por meio de pesquisas que visam conhecer as características dessa parcela populacional, evidenciando as suas especificidades e contribuindo, portanto, para a qualidade na assistência prestada aos pacientes críticos. Além disso, o conhecimento do perfil de idosas em pós-operatório, internadas no CTI, facilita a administração dos recursos e das demandas diferenciadas de cuidados.

Diante do exposto, o objetivo do estudo foi identificar o perfil clínico de mulheres idosas em pós-operatório, internadas no Centro de Terapia Intensiva de um hospital público de ensino de Belo Horizonte.

\section{Metodologia}

Trata-se de um estudo epidemiológico, com delineamento transversal, realizado no CTI de um hospital público de ensino de Belo Horizonte. Foram incluídas as mulheres com 60 anos ou mais, completados até a data da internação, provenientes do bloco cirúrgico $(B C)$ e que foram 
admitidas no CTI, entre janeiro e agosto de 2015. Foram excluídos da amostra idosos do sexo masculino e mulheres idosas provenientes de outros setores. A Figura 1 refere-se ao fluxograma das exclusões amostrais deste estudo.

O CTI no qual foi realizado o estudo é dividido em três unidades, cada uma composta por 10 leitos, e atende, em média, 45 idosos por mês.

A coleta de dados se deu por meio de consulta ao Prontuário Eletrônico (PE) das pacientes. Nos casos em que houve mais de uma admissão no CTI por paciente no referido período, foi analisada apenas a primeira internação.

As variáveis estudadas foram relacionadas ao perfil sociodemográfico (idade e cidade de residência) e internação (tempo de internação, causa da admissão, presença de lesão por pressão - LPP -, procedência e evolução do caso).

Os dados foram analisados com o auxílio do Software Statistical Software (Stata), versão 14.0 (Stata Corp., Texas, USA). A média e o desvio padrão (DP) foram calculados para as variáveis quantitativas e as frequências e as proporções foram calculadas para as variáveis categóricas. Foram calculados intervalos de confiança (IC95\%) para as proporções. Para verificar-se a associação entre o setor de procedência (BC e outros setores), a presença de UP e a resolubilidade do caso (alta melhorada, óbito e transferência para outro setores) utilizou-se o teste Qui-quadrado de Pearson. Para todos os procedimentos analíticos adotou-se o nível de significância de $5 \%$. O estudo foi submetido e aprovado pelo Comitê de Ética em Pesquisa em Saúde da Universidade Federal de Minas Gerais, sob CAAE 15690813.2.0000.5149 e número de parecer 462.743.

Figura 1 - Fluxograma do estudo. Belo Horizonte - MG - 2015.

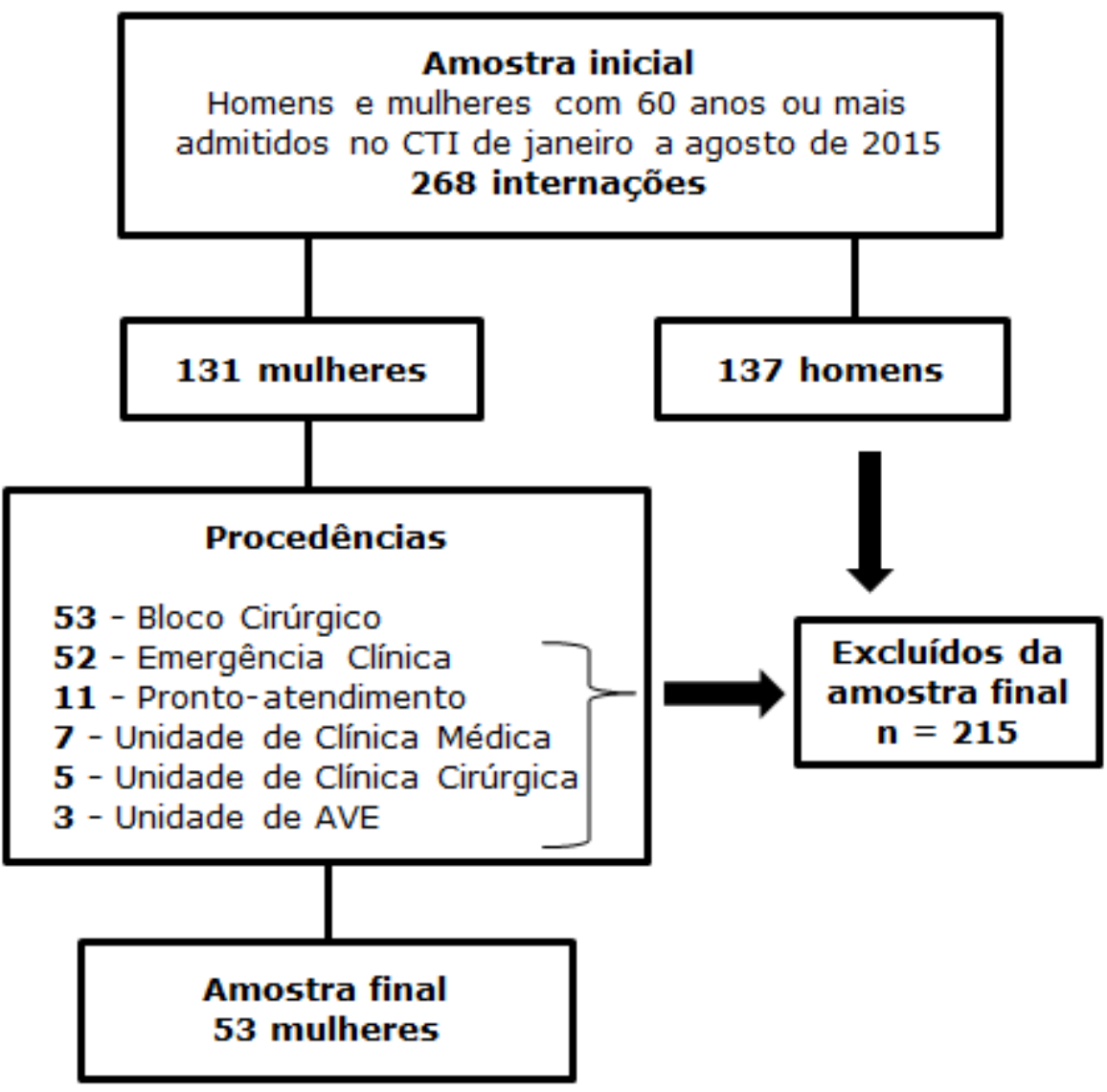

Fonte: Elaborada para fins deste estudo. 


\section{Resultados}

A amostra estudada constou de 53 mulheres idosas em pós-operatório internadas no CTI e provenientes do BC.

A idade das mulheres idosas variou entre 61 e 100 anos, com média de idade das idosas foi de
78,9 anos (DP $\pm 10,6$ anos), predomínio da faixa etária de 80 anos ou mais (47,2\%). As pacientes eram provenientes, predominantemente, da cidade de Belo Horizonte $(68,1 \%)$ (Tabela 1$)$.

Tabela 1 - Perfil Sociodemográfico da amostra. Belo Horizonte - MG - 2015.

\begin{tabular}{lccc}
\hline \multicolumn{1}{c}{ Perfil Sociodemográfico } & $\mathbf{n}$ & $\%$ & IC 95\% \\
& & & \\
\hline Cidade de procedência & 30 & 68 & $53,8-82,5$ \\
Belo Horizonte & 6 & 14 & $3,0-24,2$ \\
Santa Luzia & 2 & 4,5 & $1,9-10,9$ \\
Ribeirão das Neves & 1 & 2,3 & $2,3-6,8$ \\
Pedro Leopoldo & 1 & 2,3 & $2,3-6,8$ \\
Itabirito & 1 & 2,3 & $2,3-6,8$ \\
Vespasiano & 1 & 2,3 & $2,3-6,8$ \\
Passos & 1 & 2,3 & $2,3-6,8$ \\
Sabará & 1 & 2,3 & $2,3-6,8$ \\
Matozinhos & 44 & 100 & \\
Total & & & \\
Faixa etária (anos) & & & \\
& 13 & 25 & $12,5-36,5$ \\
60 - 69 & 15 & 28 & $15,7-40,8$ \\
70 - 79 & 15 & 28 & $15,7-40,8$ \\
80 - 89 & 53 & 100 & $7,9-29,7$ \\
90 ou mais & & & \\
Total & 10 & &
\end{tabular}

Fonte: Elaborada para fins deste estudo.

Observou-se que a duração da internação variou de 1 a 38 dias, com média de 6 dias $( \pm 7,8)$. Uma das pacientes faleceu antes de completar 24 horas de internação no CTI. Em relação às causas da admissão hospitalar, $32,1 \%$ das idosas foi internada devido à fratura, sendo predominante a de localização pertrocantérica (35,3\%) (Tabela 2).

As doenças cardiovasculares representaram a segunda maior causa de admissão e corresponderam a $24,5 \%$ do total de internação. Das idosas hospitalizadas por esses agravos,
$38,5 \%$ foram admitidas com embolia e trombose. Dentre as admitidas no CTI por causas neurológicas, 66,7\% delas foi devido ao Acidente Vascular Encefálico (AVE) (Tabela 2). Nenhuma das pacientes teve mais de uma causa de admissão relatadas no PE como causa principal da internação.

Ressalta-se que $20,9 \%$ das idosas internadas no CTI após cirurgia possuíam lesão por pressão, com diferença estatisticamente significativa $(p<0,05)$, segundo os setores de procedência (BC e outros setores) (Tabela 3). 
Tabela 2 - Dados do período de internação - Belo Horizonte - MG - 2015.

Dados do período de internaçãa

Fratura

Pertrocantérica

Colo do Fêmur

Diáfise de fêmur

Subtrocantérica

Fêmur

Ulnar

Doença Cardiovascular

Embolia e Trombose

Aterosclerose

ICC descompensada

Aneurisma de aorta abdominal

Gangrena

Doença vascular não especificada

Doença Neurológica

Acidente vascular encefálico

Doença neurológica não especificada

Doença Infecciosa

Abdômen agudo

Doença Gastroinstetinal

\section{Choque}

\section{Doença Respiratória}

Pneumonia

Total

Tempo de internação (dias)
1 a 10
11 a 20
21 a 30
31 a 38
Total

IC 95\%

3

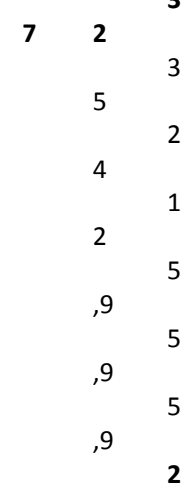

35

93

1

,6

$\begin{array}{ll}, 6 & \\ & 7\end{array}$

, 6

7.6

7

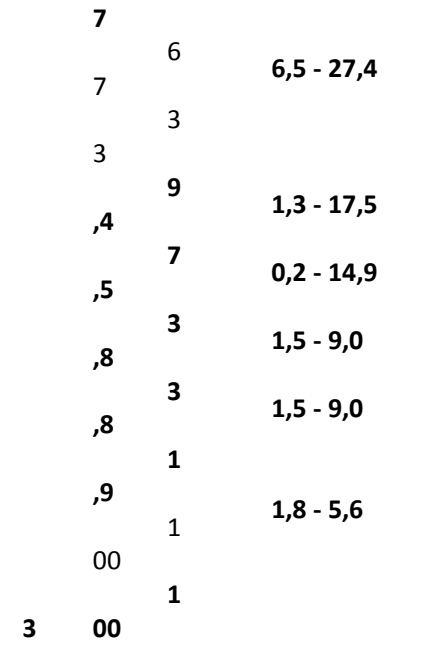

$19,0-45,0$

$12,5-36,5$ 
Em relação à evolução do caso, $73,6 \%$ das idosas receberam alta melhorada do CTI para outros setores, $22,6 \%$ evoluíram para óbito e $3,7 \%$ foram transferidas para outro hospital.
Observou-se diferença estatística $(p<0,05)$ entre óbito e alta melhorada de acordo com os setores de procedência (Gráfico 1).

Tabela 3 - Lesāo por pressão segundo setores de procedência. Belo Horizonte - MG - 2015.

\begin{tabular}{cccc}
\hline \multicolumn{3}{c}{ Procedência } \\
UP no CTI & Bloco cirúrgico & Outros setores & \\
Não & & & Total \\
$n$ & 34 & 25 & 59 \\
$\%$ & 79,07 & 39,68 & 55,66 \\
Sim & & & \\
n & 9 & 38 & 47 \\
$\%$ & 20,93 & 60,32 & 44,34 \\
Total & & & 106 \\
$\mathrm{n}$ & 43 & 63 & 100 \\
$\%$ & 100 & 100 &
\end{tabular}

Fonte: Elaborada para fins deste estudo.

Nota: $p=0,000$ (Qui-quadrado de Pearson).

Gráfico 1 - Desfecho das idosas da amostra. Belo Horizonte - MG - 2015.

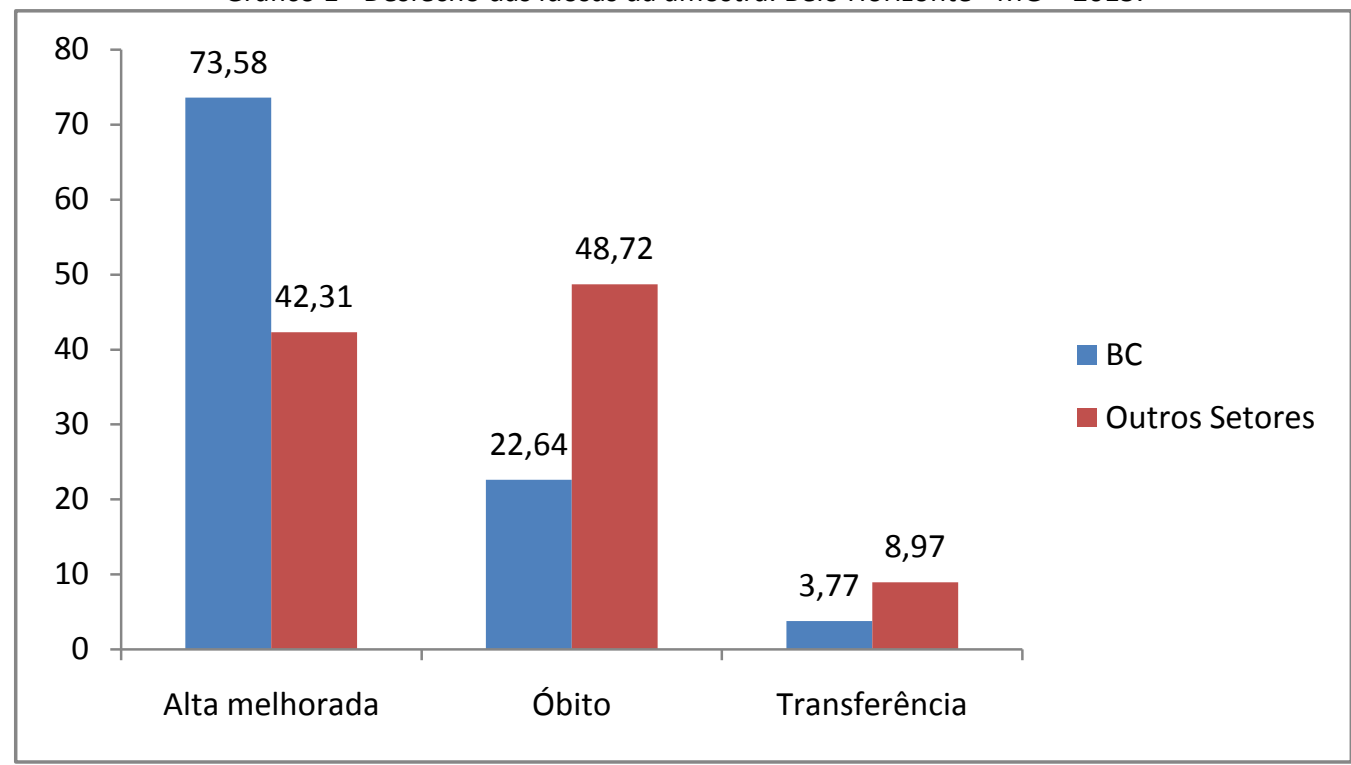

Fonte: Elaborado para fins deste estudo.

Notas: $p=0,000 ; 0,003$ e 0,248 para alta do CTI, óbito e transferência, respectivamente (Qui-quadrado de Pearson).

\section{Resultados e Discussão}

Os resultados deste estudo são corroborados por outras pesquisas sobre a análise do perfil de idosas após cirurgia, internadas no CTI. Sabe-se que a população idosa utiliza os serviços hospitalares de maneira mais intensa que os demais grupos etários, implicando em maiores custos, duração do tratamento superior e recuperação mais lenta ${ }^{12}$. Sabe-se que as internações da população idosa são mais custosas para o serviço de saúde quando 
comparadas com a população adulta. As complicações de saúde associadas ao próprio processo de envelhecimento inerente do paciente, além das doenças crônicas e as possíveis falhas nas ações de promoção da saúde e de prevenção de agravos na fase adulta como as principais causas dos gastos ${ }^{7}$.

As mulheres apresentam maior longevidade que os homens, o que as levam a períodos mais longos de doenças crônicas. Além disso, tem menor exposição a fatores de risco relacionados ao ambiente de trabalho, menor prevalência de tabagismo e uso de álcool e maior cobertura na assistência gineco-obstétrica ${ }^{13}$.

Os principais motivos que ocasionam a hospitalização do idoso em terapia intensiva são o pós-operatório imediato ou tardio de cirurgias de emergência ou grandes cirurgias eletivas, afecções cardiovasculares, doenças respiratórias e traumas ${ }^{14}$.

Em relação à média de duração da internação, o resultado desse trabalho corrobora com o relatado pelo 20 Censo Brasileiro de Unidade de Terapia Intensiva (UTI), que é de 1 a 6 dias $^{14}$. Em outra análise, a média de permanência dos idosos foi de 8,5 dias $( \pm 10,1)^{15}$. Ressalta-se que, com o aperfeiçoamento continuado de novas tecnologias, o paciente gravemente enfermo é mantido por um período prolongado nas unidades de terapia intensiva, mesmo quando a morte é inevitável, ocasionando altos custos financeiros, morais e psicológicos para todos os envolvidos ${ }^{15}$.

Neste estudo, a maioria das idosas admitidas na terapia intensiva provenientes do bloco cirúrgico foi em decorrência de fraturas. Nos idosos, as fraturas estão altamente relacionadas com a osteoporose, que acomete a ambos os sexos, mas é mais frequente na mulher, pois na pós-menopausa ocorre uma diminuição acelerada da massa óssea devido ao hipoestrogenismo ${ }^{16}$.

Associada à osteoporose, a queda e a instabilidade nos idosos contribuem para a ocorrência de fraturas. Cerca de 30\% dos idosos caem a cada ano. Essa taxa aumenta para $40 \%$ entre os idosos com mais de 80 anos e as mulheres tendem a cair mais que os homens até os 75 anos de idade ${ }^{16}$. Dos idosos que caem, cerca de 2,5\% serão hospitalizados e, desses, apenas metade sobreviverá após um ano (estimativas). Dentre os fatores de risco intrínsecos ao avançar da idade, que contribuem para as quedas, estão a idade acima de 80 anos, o sexo feminino, a imobilidade, as quedas precedentes, o equilíbrio diminuído, a marcha lenta, a baixa aptidão física, a fraqueza muscular e as alterações cognitivas ${ }^{16}$.

Segundo a localização anatômica, os principais locais fraturados pelos idosos deste trabalho foram o fêmur e quadril. Em uma pesquisa realizada em um hospital público no Rio de Janeiro, com amostra de 82 pacientes, 63,41\% apresentaram fraturas transtroncaterianas, $35,37 \%$ do colo e $1,22 \%$ subtroncateriana. Estes autores ressaltam, ainda, que as fraturas do fêmur proximal são as mais graves fraturas no idoso, requerem hospitalização e tratamento cirúrgico, em sua maioria, e apresentam altos índices de morbidade e letalidade ${ }^{17}$.

As causas cardiovasculares também merecem destaque neste estudo. É citado na literatura que na faixa etária acima de 60 anos observa-se uma alta incidência de doenças crônicas, que, quando agudizadas, levam ao adoecimento e maior uso da UTI, com as doenças cardiovasculares representando a maior causa de morte entre os idosos ${ }^{13,18}$.

Nesta pesquisa, mais de $20 \%$ das idosas em pós-operatório internadas na terapia intensiva possuíam LPP, com diferenças estatisticamente significativas para as pacientes internadas na terapia intensiva segundo setores de procedência. Essa menor proporção de LPP nas idosas provenientes do BC se deve, provavelmente, à menor prevalência de fatores de risco para o seu desenvolvimento, como imobilidade, desnutrição, edema, vasoconstrição medicamentosa, alterações do nível de consciência, incontinências e vasculopatias ${ }^{19}$.

Salienta-se que, com o avançar da idade, a pele diminui a elasticidade, a textura, a circulação, o nível de reposição celular, o processo de cicatrização da pele e a sensibilidade periférica, levando ao aumento do risco de trauma tegumentar ${ }^{20}$. Assim, os idosos tornam-se mais susceptíveis a apresentarem LPP, que é uma complicação frequente em pacientes graves e tem grande impacto sobre sua recuperação e qualidade de vida, prolongando o tempo e o custo da internação ${ }^{21}$.

Estudo realizado em uma terapia intensiva de hospital público constatou que a maioria dos pacientes $(38,1 \%)$ que apresentaram LPP encontrava-se na faixa etária de 51 anos ou mais, e a maioria $(57,1 \%)$ era do sexo feminino ${ }^{21}$.

Nesta pesquisa, $73,6 \%$ das idosas receberam alta melhorada para outros setores, $22,6 \%$ evoluíram para óbito e 3,7\% foram transferidas. Com diferenças estatisticamente significativas, a maior proporção de alta e a menor proporção de óbitos foi das idosas provenientes do BC. Isso se deve, provavelmente, 
à menor gravidade dos agravos, à menor prevalência de comorbidades e ao melhor estado geral destes indivíduos em relação aos provenientes de outros setores. Alguns autores encontraram taxas superiores aos resultados deste trabalho, sendo que a taxa de alta ou transferência dos idosos foi de $54,9 \%$ e de óbitos foi de $45,1 \%{ }^{13}$.

Em geral, quanto maior a idade, maior a ocorrência de óbito $^{13}$. A idade permanece como o maior preditor de mortalidade entre idosos, duplicando o risco de morte por qualquer causa a partir dos 70 anos $^{22}$. Estudo mostra, ainda, que a terapia intensiva é local de maior ocorrência de eventos adversos e que os idosos são os mais atingidos, levando ao aumento no tempo de internação, às lesões permanentes e ao aumento no número de óbitos ${ }^{23}$.

Os achados deste trabalho reforçam a importância de mais pesquisas voltadas para esse grupo populacional, da atuação da equipe multiprofissional e interdisciplinar e dos cuidados de enfermagem na promoção da saúde, prevenção de agravos e acompanhamento desses pacientes, de forma a reduzir a morbimortalidade e proporcionar melhor qualidade de vida aos mesmos.

Apesar dos avanços, este estudo apresenta algumas limitações, como o fato de a amostra não ser representativa para a população idosa brasileira. Ressalta-se, ainda, a perda de algumas informações para as variáveis estudadas nos prontuários eletrônicos.

\section{Conclusão}

Nesse estudo observa-se que, mulheres idosas provenientes do bloco cirúrgico internadas no CTI demonstraram melhores taxas de altas melhoradas e menor número de lesões por pressão quando comparadas com idosas provenientes de outros setores internadas no CTI.

Em virtude do aumento proporcional da população idosa em nosso País, recomenda-se aos formadores de ações em saúde e recursos humanos a adoção de políticas que possibilitem o atendimento adequado à saúde dessa população em todos os níveis de atenção à saúde, inclusive em setores específicos, como a terapia intensiva.

Ao longo dos últimos anos, avanços foram realizados no que diz respeito à saúde das mulheres. Salienta-se, contudo, que os estudos sobre as mulheres idosas ainda são escassos, principalmente sobre sua realidade, perfil e necessidades.
Desse modo, mesmo onde existe maior progresso, há a necessidade de melhorar investir em políticas públicas voltadas para as mulheres idosas, garantindo serviços de promoção da saúde, prevenção de agravos e de assistência à saúde integral.

\section{Referências}

1. Vanzella E, Nascimento JA do, Santos SR dos. $O$ envelhecimento, a transição epidemiológica da população brasileira e o impacto nas hospitalizações. Rev Elet Estácio Saúde. 2018;7(1).

2. Ervatti LR, Borges GM, Jardim A de P. Mudança Demográfica no Brasil no início do Século XXI. Subsidios para as Projeções da População. IBGE, Instituto Brasileiro de Geografia e Estatística. 2015. 1-156 p.

3. World Health Organization. Envelhecimento ativo: uma política de saude. 2005;380(9838):247-57. Available from: http://scholar.google.com/scholar?hl=en\&btnG= Search\&q=intitle:Envelhecimento+ativo:+uma+p ol?tica+de+sa?de\#0\%5Cnhttp://dx.doi.org/10.10 16/S0140-6736(12)60646-

1\%5Cnhttp://dx.doi.org/10.1016/S0140-

6736(12)61031-9\%5Cnhttp://www.aspea.org/XIV $\mathrm{J}$ - actividade fi

4. IBGE. Censo demográfico 2010: características gerais da população, religião e pessoas com deficiência. 2010;1-30.

5. Küchemann BA. Envelhecimento populacional, cuidado e cidadania: velhos dilemas e novos desafios. Soc e Estado [Internet]. 2012;27(1):165-80. Available from: http://www.scielo.br/scielo.php?script=sci_artte $\mathrm{xt} \& \mathrm{pid}=\mathrm{S} 0102$ -

69922012000100010\&lng=pt\&tlng=pt

6. Moura MAV, Domingos AM, Rassy MEC. A qualidade na atenção à saúde da mulher idosa: um relato de experiência. Rev Esc Anna Nery. 2010;14(4):848-55.

7. Silveira RE, Santos ÁS, Sousa MC, Monteiro TSA. Gastos relacionados a hospitalizações de idosos no Brasil: perspectivas de uma década. Einstein (São Paulo) [Internet]. 2013;11(34):514-20. Available from: http://www.scielo.br/scielo.php?script=sci_artte xt\&pid=S1679-45082013000400019\&lang=pt

8. Berlezi EM, Farias AM, Dallazen $F$, Oliveira KR, Pillatt AP, Fortes CK. Como está a capacidade funcional de idosos residentes em comunidades com taxa de envelhecimento populacional acelerado? Rev Bras Geriatr e Gerontol [Internet]. 2016;19(4):643-52. Available from: 
http://www.scielo.br/scielo.php?script=sci_artte xt\&pid=S1809-

98232016000400643\&lng=en\&tlng=en

9. Rabelo LP de O, Vieira MA, Caldeira AP, Costa SM. Perfil de idosos internados em um hospital universitário. REME, Rev Min Enfermagem, Belo Horiz. 2010;14(3):293-300.

10. Kotekewis K. Prevaoência de doenças crônicas não transmissíveis em idosos submetidos a procedimentos cirùrgicos [Internet]. 2013. Available from: http://www.lume.ufrgs.br/handle/10183/78409

11. Gomes GB, Gomes VR. Perfil do idoso internado em UTI do interior paulista. Rev Cienti do UNIFAE. 2012;5(2):41-6.

12. Rocha MS, Caetano JÁ, Soares E, Medeiros FL. Caracterização da população atendida em unidade de terapia intensiva: subsídio para a assistência. Rev enferm UERJ. 2007;15(3):411-6.

13. Pilger $\mathrm{C}$, Menon $\mathrm{MH}$, Mathias TA de F. Características sociodemográficas e de saúde de idosos: contribuições para os serviços de saúde. Rev Latino-Americana Enfermagem, Ribeirão Preto [Internet]. 2011;19(5):1230-8. Available from:

http://www.scielo.br/scielo.php?script=sci_artte

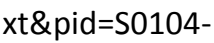

$11692011000500022 \& \operatorname{lng}=e n \& n r m=i s o \&$ tlng=pt 14. Franco LG, Kindermann AL, Tramujas L, Kock $\mathrm{K}$ de $\mathrm{S}$. Fatores associados à mortalidade em idosos hospitalizados por fraturas de fêmur. Rev Bras Ortop [Internet]. 2016;51(5):509-14. Available from: http://dx.doi.org/10.1016/j.rbo.2015.10.009

15. Schein LEC, Cesar JA. Perfil de idosos admitidos em unidades de terapia intensiva gerais em Rio Grande, RS: resultados de um estudo de demanda. Rev Bras Epidemiol [Internet]. 2010;13(2):289-301. Available from: http://www.scielo.br/pdf/rbepid/v13n2/11.pdf Brasil. Ministério da Saúde. Secretaria de Atenção à Saúde. Departamento de Atenção Básica. Envelhecimento e saúde da pessoa idosa. Brasília, 2007.

16. Fernandes RA, Araújo DV, Takemoto MLS, Sauberman MV. Fraturas do fêmur proximal no idoso: estudo de custo da doença sob a perspectiva de um hospital público no Rio de Janeiro, Brasil. Physis, 2011; 21(2): 395-416.

17. Piuvezam G, Medeiros WR, Costa AV, Emereciano FF, Santos RC, Seabra DS. Mortalidade em idosos por doenças cardiovasculares: análise comparativa de dois quinquênios. Arq bras cardiol [online], 2015. Disponível http://www.scielo.br/pdf/abc/2015nahead/pt_0 066-782X-abc-20150096.pdf.

18. D'arco C, Sassine SW, Costa MLM, Silva LMG. Úlcera de pressão em UTI. In: Knobel E. Condutas no paciente grave. 3a. ed. São Paulo: Atheneu; 2006. Vol. 2. p.2491-501.

19. Pieper B. Mechanical forces: pressure, shear, and friction. In: Bryant RA, Nix DP. Acute and chronic wounds: current management concepts. 3rd ed. St. Louis: Mosby, 2007. p. 20534.

20. Silva EWNL, Araújo RA, Oliveira EC, Falcão VTFL. Aplicabilidade do protocolo de prevenção de úlcera de pressão em unidade de terapia intensiva. Rev bras ter intensiva. 2010; 22(2): 175-85.

21. Rooji SE, Abu-Hanna A, Levi M, Jonge E. Factors that predict outcome of intensive care treatment in very elderly patients: a review. Crit care. 2005 Aug; 9(4): 307-14.

22. Pedreira LC, Brandão AS, Reis AM. Evento adverso no idoso em Unidade de Terapia Intensiva. Rev bras enferm. 2013 mai/jun; 66(3): 429-36.

\section{Endereço para Correspondência}

Universidade Federal de Minas Gerais - UFMG

Av. Pres. Antônio Carlos, 6627 - Pampulha, Belo Horizonte - MG

CEP.: $31270-901$

e-mail: latiniflavia@gmail.com

Recebido em 22/08/2017

Aprovado em 19/07/2018

Publicado em 12/09/2018 